\title{
Using Electron Backscatter Diffraction to Aid Identification of Fossilized Dinosaur Eggshells
}

\author{
P.W. Trimby, ${ }^{1}$ and G. Grellet-Tinner ${ }^{2,3,4}$ \\ ${ }^{1}$ The Australian Centre for Microscopy \& Microanalysis, \\ The University of Sydney, NSW 2006, Australia \\ 2 The Field Museum, Chicago, Illinois 60605, ${ }^{3}$ The Journey Museum, Rapid City, South Dakota \\ $57701,{ }^{4}$ Investigador Correspondiente of CONICET, Argentina
}

For a number of years microscopy techniques have been extensively used to help with the identification of fossilized dinosaur eggshells [1]. Morphological parameters such as the eggshell thickness, the pore size and spacing, the number of discrete layers in the cross section and so on allow paleontologists to identify the broad phylogenetic group to which individual eggshells are assigned. Most of these measurements involve the study of eggshell sections using light microscopy techniques and by imaging in a scanning electron microscope (SEM). Occasionally energy dispersive X-ray spectroscopy (EDS) is necessary to distinguish between late stage diagenetic infilling or alteration (usually involving silicate minerals) and the original fossilized structure (usually calcite, $\mathrm{CaCO}_{3}$ ). However, many of the observations are subjective and it is challenging to develop a rigorous classification scheme to distinguish between fossilized eggshells.

Electron backscatter diffraction (EBSD) in the SEM has been successfully used to characterize extant avian eggshells [2], as well as to study other fossilized structures, including trilobite eyes [3] and seashells [4]. EBSD has a significant advantage over other microscopy techniques in that it enables the crystalline structure (and hence the growth patterns) of the eggshell to be quantified, both in terms of spatial distribution and in terms of lattice preferred orientation (LPO) or texture. In this paper we present some of the first results of EBSD analyses from a range of fossilized eggshells, both from dinosaurs and from non-dinosaur reptiles and birds.

The EBSD measurements were collected using automated orientation mapping across the polished surface of the cross section through the eggshells. The spacing between individual measurements ranged from 0.5 to 2 micrometers, and most datasets contained more than 1 million analyzed points. The results enable us to highlight and measure many parameters, including the following:

1. The size and spacing of individual eggshell "units". These are interpreted as originating from a single nucleation point on the inner surface of the shell, from which the calcite crystals grow in a c-axis (0001) dominated orientation. Part of an orientation map showing a single shell unit from a Neosauropod eggshell is shown in figure 1.

2. The nature and strength of any LPO (texture). This is typified by a strong c-axis alignment with the shell's radial direction, as shown in figure 2.

3. The frequency distribution of boundary misorientations in each eggshell.

4. The crystallographic signature of individual layers in eggshells (where present).

5. The nature and distribution of grain sizes within eggshells.

6. Identification of late stage diagenetic alteration, when this does not involve silicate phases.

EBSD is a perfect technique for characterizing subtle differences between fossilized eggshells. Many different parameters can be used to aid classification schemes, thereby allowing paleontologists to identify the phylogenitic groups to which certain eggshells belong. Initial results 
in this field are already making an impact [5], and it is expected that EBSD will, in the future, become a standard tool for the microanalysis of fossilized eggshells.

\section{References}

[1] G. Grellet-Tinner, Alcheringa 30 (2006), 130-180

[2] P. Dalbeck and M. Cusack, Crystal Growth and Design, 6 (2006), 2558-2562.

[3] M. R. Lee et al., Palaeontology 50 (2007), 1031-1037.

[4] A. Pérez-Huerta et al., Palaeontology 50 (2007), 757-763.

[5] G. Grellet-Tinner et al., Gondwana Research (in press)

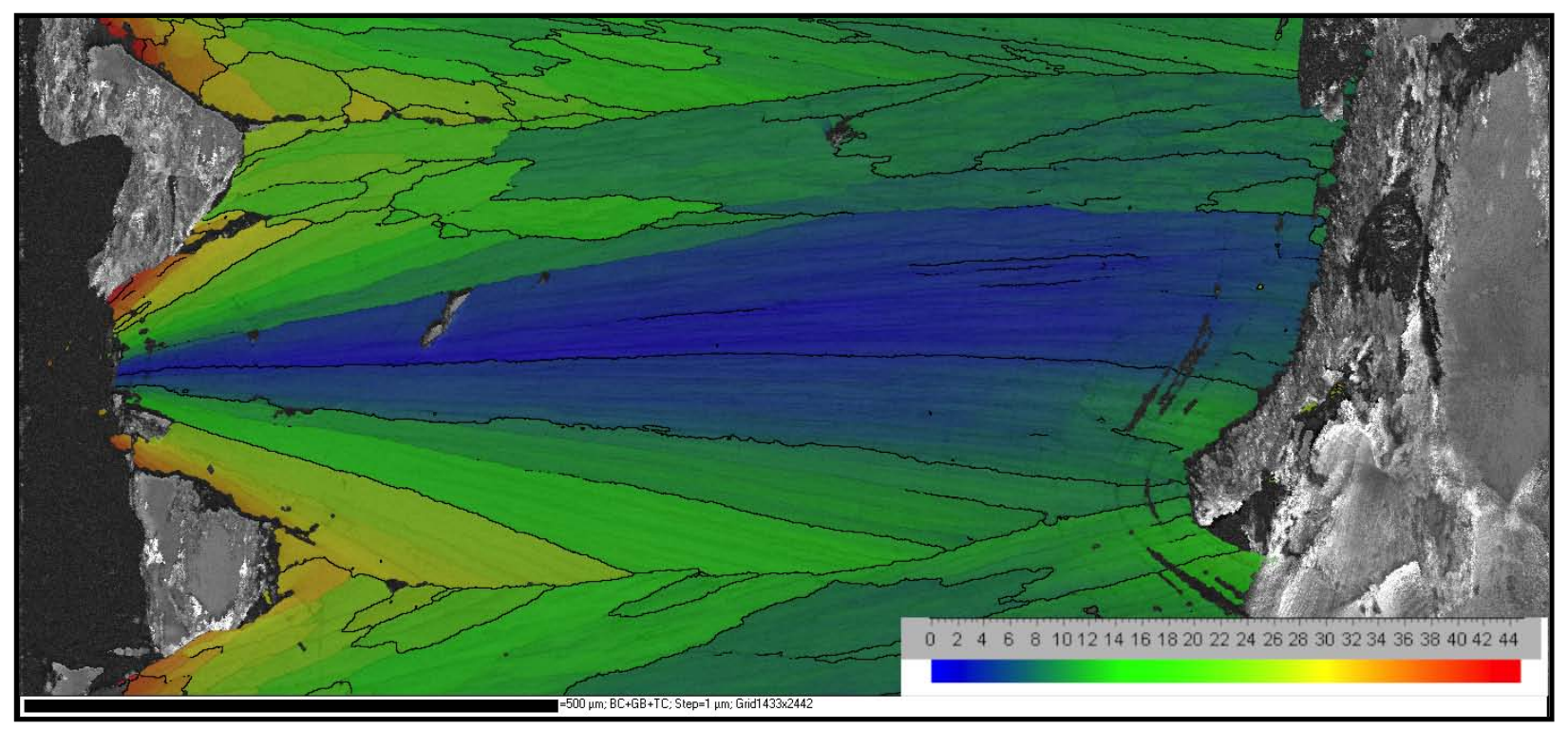

FIG. 1. Part of an EBSD orientation map showing a single shell unit in a cross section through a fossilized Neosauropod eggshell. The colors correspond to the deviation from a c-axis alignment with the eggshell's radial direction (horizontal in the map), varying from $0^{\circ}$ (blue) to $45^{\circ}$ (red), with high angle boundaries $\left(>10^{\circ}\right)$ marked with black lines. The outer surface of the egg is to the right, and the grayscale indicates regions from which no EBSD indexing was possible. The scale bar marks $500 \mu \mathrm{m}$.

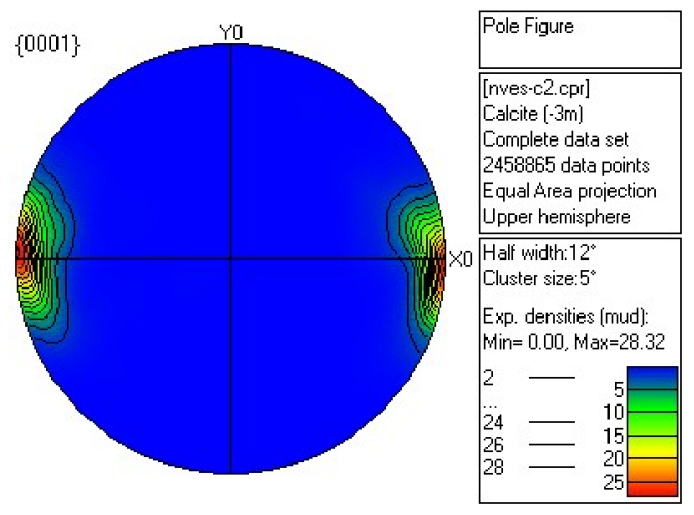

FIG. 2. Contoured c-axis (0001) pole figure from the same eggshell shown in fig. 1, showing the strong alignment of the calcite c-axes with the eggshell's radial direction (X0). 